\title{
Information measuring subsystem oil pumping station "Parabel"
}

\author{
Galina S. Nyashina, Dmitrii O. Glushkov
}

National Research Tomsk Polytechnic University, 634050 Tomsk, Russia

\begin{abstract}
Information-measurement subsystem oil pumping station (OPS) "Parabel", located on the site of the main pipeline "Alexandrov-Anzhero" (OJSC "AK" Transneft "). Developed on the basis of a modern microprocessor equipment, automation, as well as high-speed digital data channels. The simple solution to meet the requirements set out in the guidance document "Automation and remote control of trunk pipelines. «General provisions» (RD-35.240.0000-KTN-207-08).
\end{abstract}

\section{Introduction}

In connection with the development of pipeline transportation in the territory of the Russian Federation much attention is paid to the basic structural units of the main oil pipeline- oil pumping stations. On one site of the pipeline may be about ten units. The rapid provision of information on the characteristics of the technological process in one coordination center, to ensure the safe operation of expensive equipment in the optimal mode of operation. It is for this purpose created information-measuring subsystem of OPS, which is an important part of the distributed control system main pipeline.

Information-measuring subsystem includes a complex (software and hardware tools, measuring and computational converters) for obtaining information on a condition of the technological object visualization and registration of parameters in an automatic mode [1]. Also important element of information-measuring subsystem are communication channels of sensors, programmable logic controllers, servers, workstation operators, printers and other peripheral equipment.

The main task of information-measuring subsystem is to provide operational information on all parameters of a technological object in real time.

The aim of the work is the creation of information-measuring subsystem oil pumping station "Parabel that implements the functions of measurement parameters of the object, transfers of receiving information to the coordination center, reporting a convenient form.

\section{The main part}

The microprocessor automatic system OPS has a complex structure. The information-measuring subsystem may include several thousands of signals. Figure 1 is a diagram of automation of the main technological equipment- the main pumps, realized by the three-level principle.

The lower level consists of microprocessor and relay of measurement and control of technological parameters of the system (temperature, pressure, level, flow, vibration, etc.... d.). This equipment is located on the process object or next to it (in the instrument boards or racks).

The middle level is composed of control panels with programmable logic controllers. The equipment on the middle level is necessary for collection of information from sensors, a filtration, linearization and scaling of entrance analog signals, control of discrete parameters. Exchange of information between higher and lower levels of automation by means of bond equipment (switches, converters, digital communication channels).

The highest level is a hardware-software complex as a part of the server of input-output, the automated workplaces (AW) of operators with the established software (SCADA system). This level is intended for supervisory control of technological object, and also for storage of the current and archival parameters, providing reports to the staff [2]. 

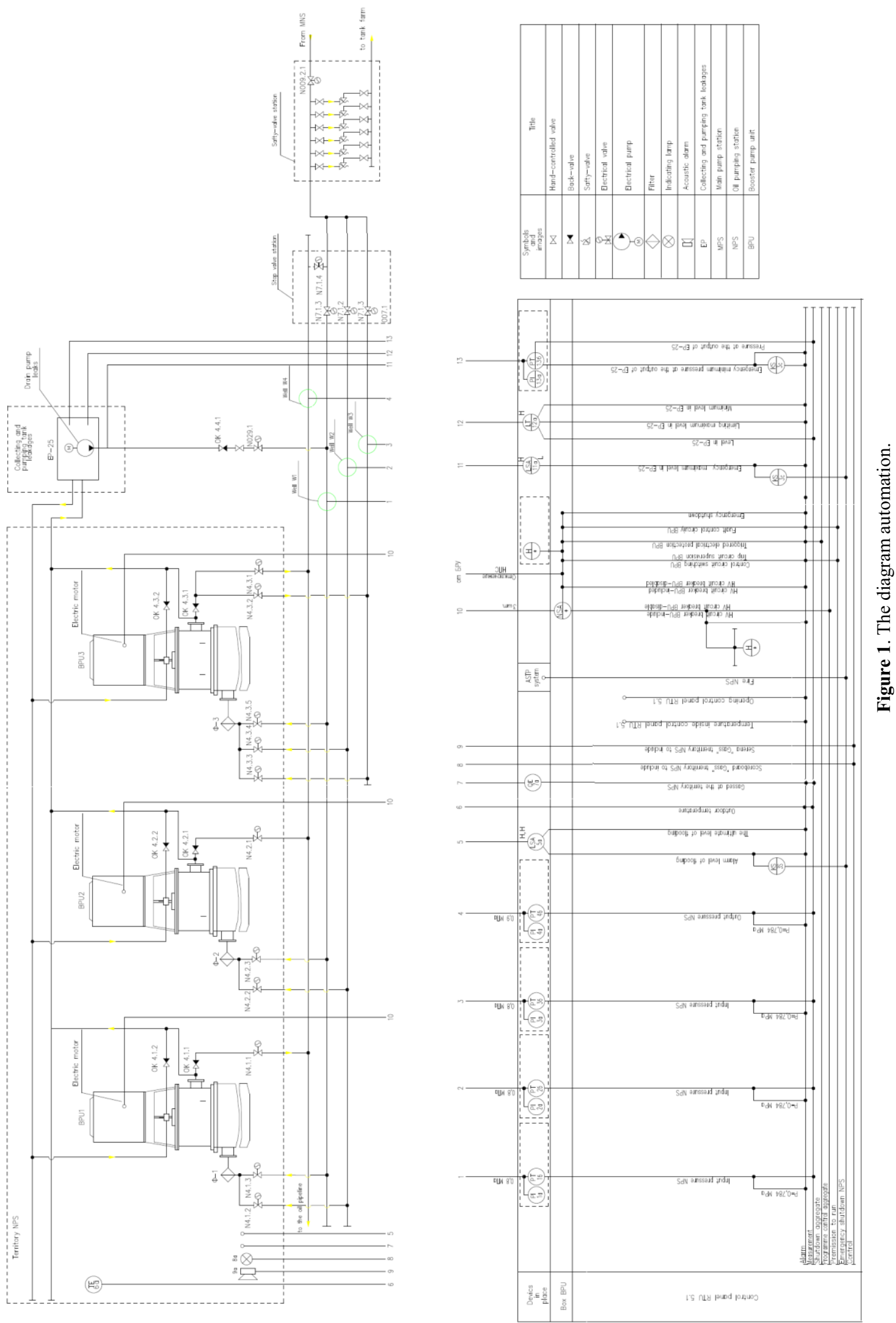

the process.

In the development of automation schemes and selection of technical means necessary to consider features of

The main requirements for lower-level equipment are relevant climatic performance (Uhl1, Uhl4) to a sensor installation site, as well as the possibility of its calibration and verification of measurement channel at the place of operation. For the middle level main requirements are an uninterrupted power supply of control panels, hot backup central controller, and also the equipment and channels of digital communication.

For the higher level necessary dual redundancy of equipment with the use of the failover server of inputoutput that communicates with all the Central controllers and the local AW of the Modbus TCP/IP protocol [3].

\section{Conclusion}

Technical solutions adopted in the implementation of information-measuring subsystem OPS "Parabel" meet the requirements of RD-35.240.00-KTN-207-08 control of process parameters, as well as the required reliability and safety data. Developed subsystem will allow the automatic control settings, collection, storage, processing and transmission of information on the process and equipment status. Multilevel implementation principle, modern equipment and automation tools, the ability to transmit information over long distances, improves the quality of the backup management.

A study performed by the Russian Science Foundation grant (project № 14-39-00003).

\section{References}

1. A.P. Ivanov, I.D. Kizina, JSC "Nefteavtomatika" on ways to improve products and to improve the quality of services in metrology and automation of oil and gas and related industries // Automation, Remote Control and Communication in the oil industry. 4, 2 (2008)

2. M.N. Bagamanov JSC "Main Oil Pipelines Central Siberia" // crude oil pipeline. 8, 54 (2008)

3. O.A. Suprun, A.S. Saifullin, V.M. Valiev, V.I. Steshin Structural and functional organization of a pumping station at "Perm-3" // Automation, Remote Control and Communication in the oil industry. 3, 68 (2006). 